\title{
Adoption of a new gait system to evaluate the clinical effects of minimally invasive needle-knife scope therapy for the treatment of rheumatoid arthritis of the knee joint
}

\author{
Zhihuang Chen ${ }^{1}$, Song Wei ${ }^{1}$, Wei Xu ${ }^{1}$, Xiaohao Li ${ }^{1}$, Xianxian Zhang ${ }^{1}$, Yinwan Liu ${ }^{1}$, Hui Li ${ }^{1}$, \\ Jianchun Chen ${ }^{2}$ \\ ${ }^{1}$ Department of Traditional Chinese Medicine, General Hospital of Southern Theater Command, PLA, Guangzhou, China; ${ }^{2}$ Chengdu Rheumatism \\ Hospital, Chengdu, China \\ Contributions: (I) Conception and design: S Wei, Z Chen; (II) Administrative support: S Wei, W Xu; (III) Provision of study materials or patients: X \\ Li, X Zhang, J Chen; (IV) Collection and assembly of data: Y Liu, J Chen; (V) Data analysis and interpretation: Z Chen, X Zhang; (VI) Manuscript \\ writing: All authors; (VII) Final approval of manuscript: All authors. \\ Correspondence to: Song Wei. Department of Traditional Chinese Medicine, General Hospital of Southern Theater Command, PLA, Guangzhou, \\ China. Email: 18665032086@163.com.
}

Background: The purpose of this study was to use kinematic gait analysis, to evaluate the clinically curative effects of minimally invasive needle-knife scope therapy in the treatment of rheumatoid arthritis (RA) of the knee joint.

Methods: A total of 32 patients with RA of the knee joint were treated with minimally invasive needleknife scope therapy. All patients were assessed with a kinematic gait analysis system, and then analyzed and compared the measurements with the kinematic gait performances of 28 healthy middle-aged and elderly participants.

Results: Before receiving the therapy, the ranges of motion (ROMs) of the patients' tibial inward and outward rotation, flexion and extension, anterior and posterior displacement of the tibia, and tibial upward and downward movement were all smaller than those of the healthy middle-aged and elderly group $(\mathrm{P}<0.05)$. After receiving the therapy, the patients' knees had a remarkably larger ROM. After one month of this therapy, they had a noticeably greater ROM in flexion and extension, tibial inward and outward rotation, and upward and downward movement of the tibia $(\mathrm{P}<0.05)$. After 1 month of therapy, the patients' maximum flexion angle and tibial posterior displacement angle were noticeably greater than beforehand $(\mathrm{P}<0.01)$. After 1 year of therapy, the patients' flexion and extension range became remarkably greater than it was after 1 month of the therapy $(\mathrm{P}<0.01)$. Their ranges of DOF reflected no apparent difference to the healthy middle-aged and elderly group, which remained smaller than the latter $(\mathrm{P}<0.05)$. After 1 year of therapy, the maximum values of the patients' flexion angle, tibial internal rotation angle, tibial posterior displacement angle, and tibial downward movement angle were remarkably higher than before commencing treatment $(\mathrm{P}<0.05)$.

Conclusions: Minimally invasive needle-knife scope therapy enables a good recovery of function for patients with RA and remarkably improves the DOF of knees. Gait analyses are more objective, accurate, and quantitative than other indexes, and thus may become a new means to assess the changes in knee joint functions.

Keywords: Rheumatoid arthritis of the knee joint; gait analysis; minimally invasive needle-knife scope; kinematic change

Submitted Jul 03, 2020. Accepted for publication Sep 11, 2020.

doi: 10.21037/apm-20-1705

View this article at: http://dx.doi.org/10.21037/apm-20-1705 


\section{Introduction}

Rheumatoid arthritis (RA) of the knee joint is an autoimmune disease which may also attack multiple joints throughout the body. It has the major clinical manifestations of chronic, symmetric, and polyarticular diseases, although its etiology and pathogenesis is not yet completely clear. The incidence rate is approximately $1 \%$ globally, about $0.3-0.4 \%$ in China, and $1-2 \%$ in Europe and the USA. The majority of patients are aged 40-60 and are female. This disease presents a severe threat to human health. In the early phase, the main clinical manifestations include red swollen patches and heat with pain in the hands, wrists, and feet along with as dysfunction of these parts. During the later stages, varying degrees of stiffness may occur in the patients' joints, which eventually leads to disability. Most patients with RA suffer from permanent irreversible destruction of the bone. Around 50\% of patients experience bone destruction and joint space narrowing within 2 years of onset, and $50 \%$ display erosion as detected by X-ray, within the first 5 years (1-3). So far there is a lack of clarity regarding the pathological process of RA. We know that bone destruction is the main cause of RA disability and that synovial lesions play a significant role in the osteoarticular damage of RA. The basic pathological change in RA is chronic inflammation of the synovium. When hyperplasia occurs in the synovium and a pannus is formed, cartilage, bones, and articular capsules can erode which results in joint deformity. Ankylosis of the joint subsequently occurs and is followed by the decline of joint functions (4).

Needle-knife scope therapy is minimally invasive, and is based on anatomy according to modern medicine, while also being guided by the tendinomuscular meridian pathway theories of traditional Chinese medicine. Using an endoscope for navigation, a small needle-knife is used to perform the surgery, stripping and loosening aponeurotic entrapments and adhesions. In accordance with holistic philosophy, the delicate and sensitive meridian regulating devices of traditional Chinese medicine have been developed to protect the overall structure when physically addressing diseases of the muscles and tendons. Thus, the needle-knife scope therapy reduces the pressure and stimulation from osteophytes, so as to clinically dredge the meridians and tendons. This therapy has been used to treat many types of rheumatic joint swelling and pain, in particular RA (5-8). Our clinical study found that this therapy can effectively reduce joint swelling, pain, and morning stiffness, improve joint function, and lower inflammatory markers including erythrocyte sedimentation rate $(\mathrm{ESR})$ and C-reactive protein (CRP) (6-8) for RA patients. Minimally invasive needle-knife scope technology has the advantage of protecting the overall structure of the body by causing microstructural changes, little damage to the tissues, and rapid recovery. The current leading methods to assess progression of and therapeutic effects for RA include joint evaluation, radiological examination, lab detection, and patient questionnaires (9). Examples of such methods are the Disease Activity Score (DAS) and Visual Analog Scale (VAS) by the American College of Rheumatology (ACR). Imaging results usually show unapparent changes within certain time periods and lack objective comparison methods; as a subjective sensation, pain is easily influenced by patient perception and environmental factors. Gait analysis is a dynamic method that has been applied to explore the dynamic and kinematic characteristics of arthritis. It can provide precise quantitative analysis for the characteristics of biomechanical changes in patients' knees in both static and dynamic conditions, thus offering a totally new method to objectively assess knee joint functions. This study uses gait analysis to assess the curative effects of minimally invasive needle-knife scope therapy in the treatment of knee joint RA, and adopts objective indexes to quantitatively analyze the changes in knee joint function before and after treatment.

We present the following article in accordance with the STROBE reporting checklist (available at http://dx.doi. org/10.21037/apm-20-1705).

\section{Methods}

\section{Patients}

This study included 32 patients with RA of the knee joint who were treated in the Department of Traditional Chinese Medicine, Southern War Zone General Hospital, from Mar 2013 to Oct 2016. The patients were diagnosed according to 1987 revised criteria for the classification of RA (2), and met the DAS and the European league against rheumatism (EULAR) response criteria (1). The inclusion criteria are as follows: (I) conformity to the above RA diagnostic criteria with moderate or above disease activity; (II) 18-70 years old; (III) at least one joint tenderness onset count $\geq 10$ and one joint swelling count $\geq 8$; (IV) CRP $\geq 2 \mathrm{mg} / \mathrm{dL}$ or ESR $\geq 28 \mathrm{~mm} / \mathrm{h}$, as detected by the Westergren method; (V) willing and able to participate fully in this research program. The study was approved by the institutional research ethics committee of the Southern War Zone General Hospital and written informed consent was obtained from all 

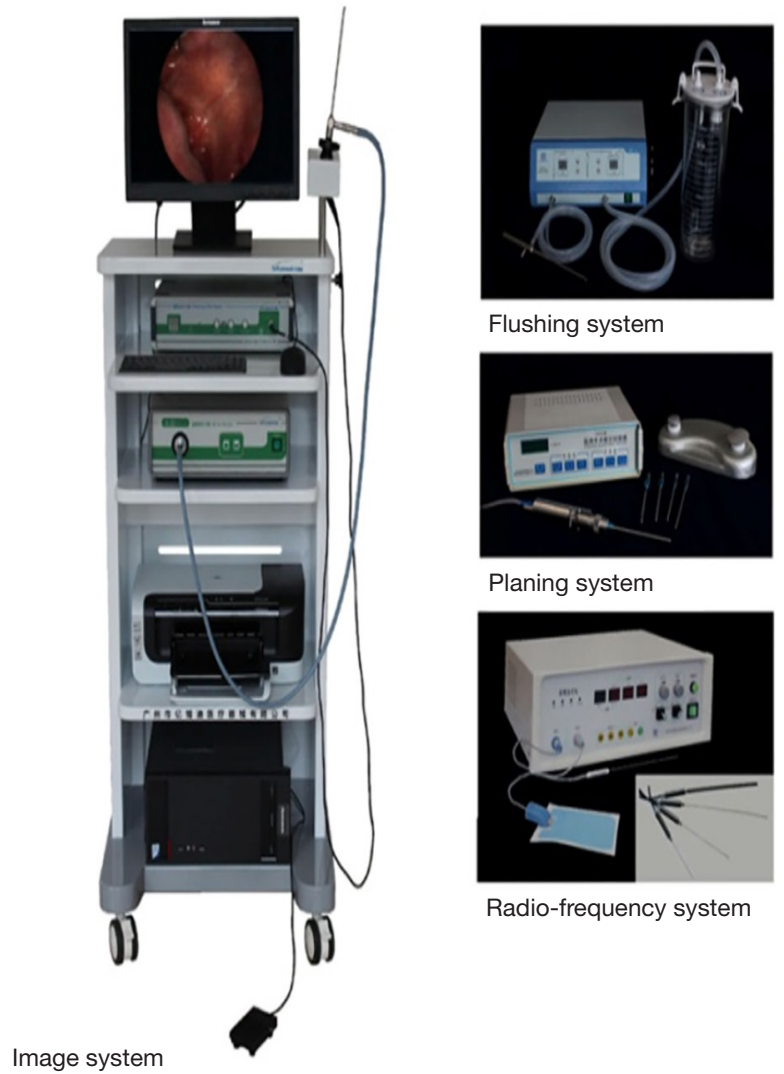

Flushing system

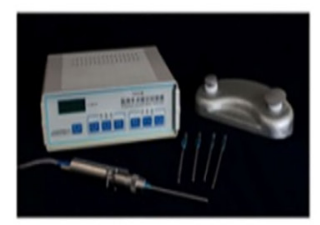

Planing system

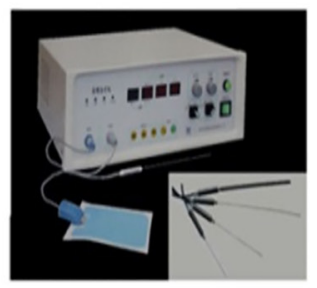

Radio-frequency system

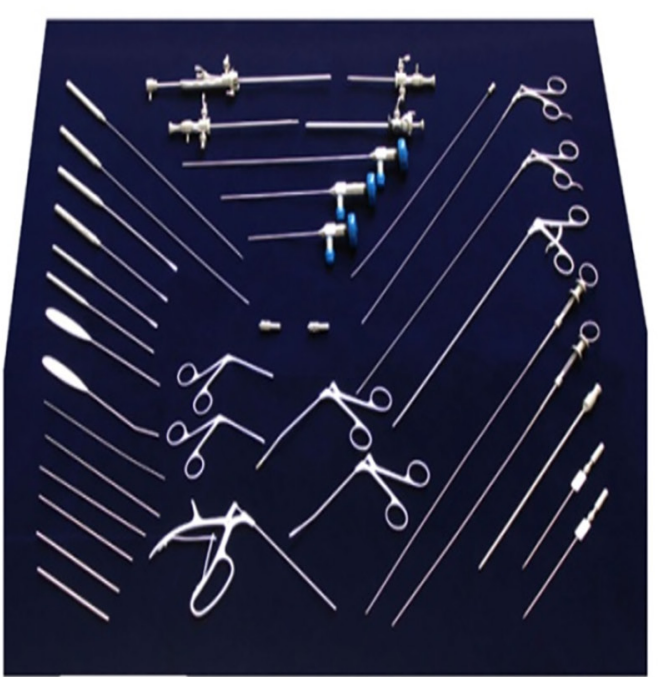

Meridian-sinew scope operation apparatus

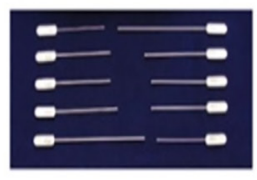

Meridian-sinew Knife

Figure 1 Minimally invasive needle-knife scope system.

participants. The estimation of sample size was based on previous study results and clinical efficacy was increased by $25 \%$ according to the estimation formula. The content of each sample was calculated following the formula $\mathrm{n}=(\mathrm{U} \alpha+$ $\mathrm{U} \beta) 22 \mathrm{P}(1-\mathrm{P}) /(\mathrm{P} 1-\mathrm{P} 0)^{2}(10)$. All procedures performed in this study involving human participants were in accordance with the Declaration of Helsinki (as revised in 2013).

\section{Intervention}

The subjects were informed before the research that this study aimed to assess the clinical curative effects of minimally invasive needle-knife scope therapy in the treatment of RA of the knee joint, and that during the process, other treatments would be suspended entirely. They were treated with methotrexate (produced by Shanghai Hualian Pharmaceutical Factory, approval number: J20120063), administered orally $10 \mathrm{mg} /$ week and celecoxib capsules (produced by Pfizer Pharmaceuticals Co., Ltd., approval number: J20120063) 0.1 g/day.
The minimally invasive needle-knife scope therapy was conducted with medical treatment.

\section{Disposal}

Minimally invasive needle-knife scope therapy applies the minimally invasive needle-knife scope system (see Figure 1), which loosens, strips, cuts and repairs pathological tissues of the knee joint with the invasive needle-knife while using an arthroscope. It is a practical technological advancement of modern Chinese medicine which was invented under the guidance of muscle meridian theory from the book Internal Classic and on the basis of the traditional "nine needles" of Chinese acupuncture. Treatment with the invasive needleknife scope lasted 4 weeks. In the first week, needle-knife were used to loosen the pathological tissues in the joint. The process was as follows: the joint entry point was located and local infiltration anesthesia with $2 \%$ lidocaine was conducted; the skin of the located point was cut with a surgical knife which then vertically stripped the tendons and 


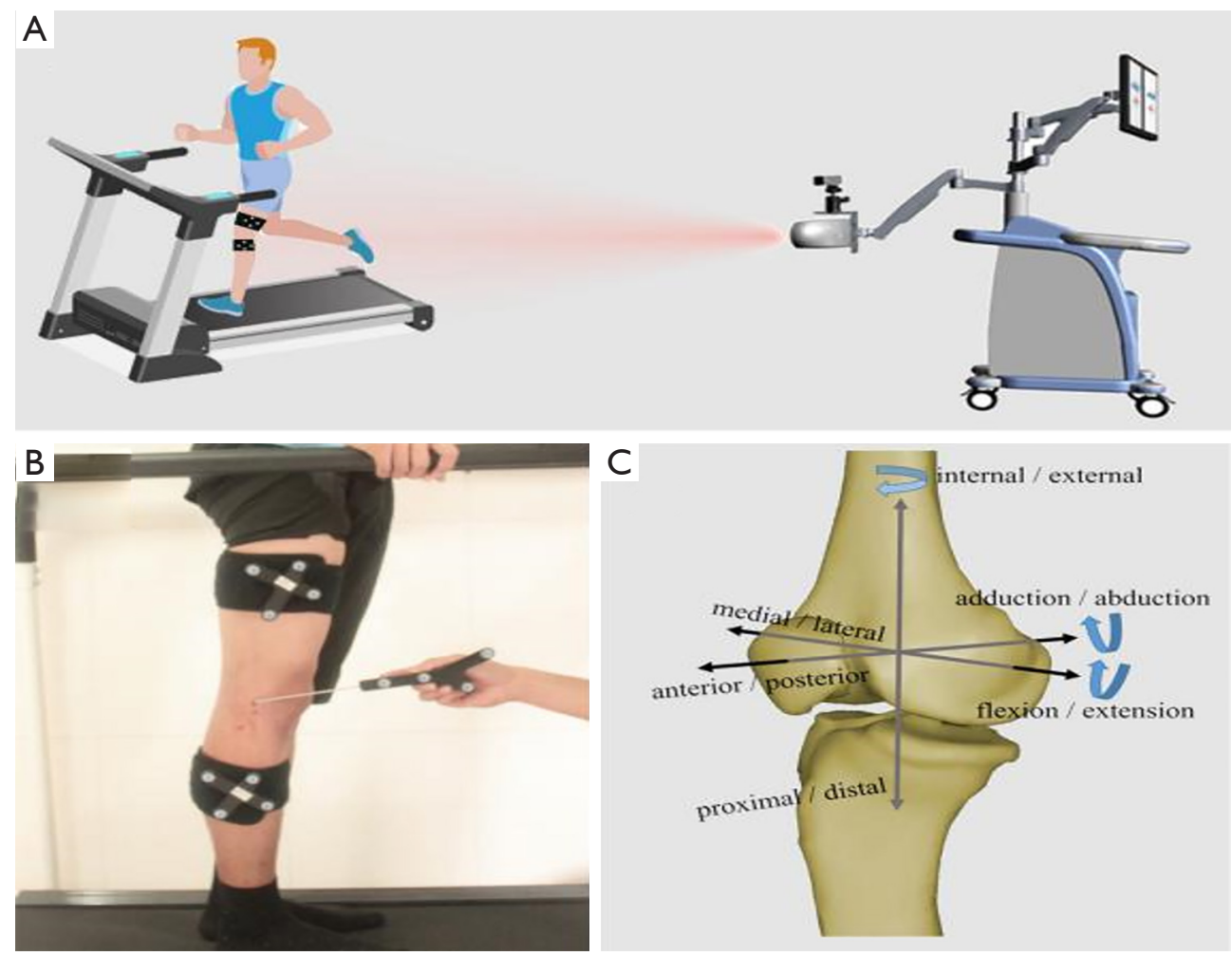

Figure 2 Principle and devices of kinematics acquisition. (A) Infrared light-navigated motion capture device; (B) spatial localization of bone markers on the body surface by virtue of infrared reflectors fastened to the distal femur and proximal tibia as well as a handheld reflective marker; (C) building a space coordinate motion system of the tibia with respect to the femur and 6 degrees of freedom.

muscle layers under skin; the joint capsule was cut to create a surgical portal for the needle-knife scope, the fit casing of needle-knife scope was imbedded, the core pulled out, and the light microscope was imbedded; the lavage access pathway was connected with the lavage injected. Then, the joint tissues and structures were carefully inspected in chronological order. When pathological tissues were spotted, loosening, stripping, repair of cartilage, removal of foreign impurities, and synovial planning were undertaken. Joint fluid drainage and lavage devices were imbedded and the joint was sustainedly lavaged until the lavage fluid was clear. The tendon knife treatment was administered after the first week. We followed the method outlined in the book Chinese Channel Tendon Theory (11) of locating transverse nodes of the tendon, cords, nodules, local tenderness, and the focal point of the fascia where local skin temperature was increased around the pathological aspect of the joint and in the medial and lateral joint space. After identifying these sites, they were marked with methylene blue, and given local infiltration anesthesia with $2 \%$ lidocaine. Tendon knives were used to vertically cut the marks through to the deep tissues and the bone surface, so as to longitudinally dredge and transversally strip the tendons. This process was repeated once a week for 3 more weeks.

\section{Gait analysis}

Infrared light navigation motion capture system (Opti Knee $^{\circledR}$, Shanghai InnoMotion, see Figure 2) was applied to analyze the participants' gaits before treatment, 1 month after treatment, and 1 year after treatment. After being directed by the doctor familiarize with the machines evaluation process, every patient walked at a normal pace bare-foot on the test platform. They were told to hold their chests and heads up with arms hung down at the sides of their bodies. Both lower limbs swang in regular and rhythmic cycles, wherein the 6 movement parameters of the knees were collected.

\section{Statistical analysis}

The Stata/SE 9.2 statistical software was used to process 
Table 1 Demographics and baseline characteristics

\begin{tabular}{lc}
\hline Parameter & $\mathrm{n}=32$ \\
\hline Age (year) & $51.0 \pm 12.9$ \\
Females, $\mathrm{n}(\%)$ & $34(85.0)$ \\
RA duration (year) & $1.0 \pm 0.6$ \\
Weight (kg) & $55.3 \pm 9.5$ \\
ESR (mm/h) & $61.1 \pm 29.8$ \\
CRP (mg/dL) & $3.0 \pm 3.1$ \\
Swollen joint count & \\
0-28 & $10.9 \pm 5.1$ \\
0-66 & $16.9 \pm 6.5$ \\
Tender joint count & \\
0-28 & $13.1 \pm 6.0$ \\
0-66 & $20.9 \pm 9.9$ \\
DAS28-ESR & $6.6 \pm 1.0$ \\
DAS28-CRP & $5.9 \pm 1.1$ \\
HAQ-DI score & $1.1 \pm 0.8$ \\
SDAl score & $40.9 \pm 12.5$ \\
CDAl score & $38.1 \pm 11.5$ \\
activity (mm) & $65.2 \pm 24.1$ \\
\hline Datient's global assessment of disease & \\
\hline
\end{tabular}

Data are mean \pm SD unless otherwise indicated. CDAI, clinical disease activity index; CRP, $C$ reactive protein; DAS28-CRP, disease activity score using a 28-joint count and CRP level;DAS28ESR, disease activity score using a 28-joint count and ESR; ESR, erythrocyte sedimentation rate; HAQ-DI, Health Assessment Questionnaire disability index; SDAI, simplified disease activity index.

data. Differences between the treatment groups in ACR response rates were assessed using a Chi-squared $\left(\chi^{2}\right)$ test. Secondary endpoints were analyzed using the Fisher's exact test and Wilcoxon rank sum test for discrete variables and continuous variables, respectively. Univariate logistic regression analysis was performed, applying 14 baseline demographics and disease characteristics. The difference in testing was considered statistically significant due to $\mathrm{P}<0.05$.

\section{Results}

The subjects' demographic characteristics and anthropometric features were collected and are shown in Table 1. Figure 3 shows the mean and standard deviation of the participants' 6 degrees of freedom while walking on the treadmill (the femur relative to the tibia) and Figure 4 shows the change in degrees of movement of the joint during walking.

Before the therapy, the ROM of the patients' tibial inward and outward rotation, flexion and extension, tibial anterior and posterior displacement, and tibial upward and downward movement were all smaller than those of the healthy middle-aged and elderly group $(\mathrm{P}<0.05)$. After having received the therapy, the patients' knees had a remarkably greater ROM. After 1 month of the therapy, they had a noticeably greater ROM in flexion and extension, inward and outward rotation of the tibia, and tibial upward and downward movement $(\mathrm{P}<0.05)$, but the ROMs of their flexion and extension and tibial anterior and posterior displacement were still smaller than those of the healthy control group $(\mathrm{P}<0.05)$. At follow up 1year after the therapy, the participants' flexion and extension range became remarkably wider than that after 1 month of the therapy $(\mathrm{P}<0.01)$. Their ranges of DOF reflected no apparent difference from the healthy middle-aged and elderly group except that their anterior and posterior tibial displacement range remained smaller than those of the control group $(\mathrm{P}<0.05)$.

While in the swing phase, the maximum values of flexion angle, tibial inward rotation angle, tibial posterior displacement angle, and tibial downward movement angle of the patients were apparently lower than those of the healthy group $(\mathrm{P}<0.01)$. After having undergone the therapy for 1 month, the participants' maximum angle of flexion and tibial posterior displacement angle were noticeably larger than before starting therapy $(\mathrm{P}<0.01)$. However, their maximum flexion angle, tibial posterior displacement angle, and tibial downward movement angle were still smaller than the healthy group's $(\mathrm{P}<0.05)$ after 1 month of therapy. After 1 year of having had the therapy, the maximum values of the patients' flexion angle, tibial inward rotation angle, tibial posterior displacement angle, and tibial downward movement angle of the patients were remarkably higher than before the treatment $(\mathrm{P}<0.05)$, with the maximum value of their flexion angle apparently higher than 1 month after the treatment $(\mathrm{P}<0.01)$. However, compared to the control group, the maximum value of the patients' angle of flexion was still lower $(\mathrm{P}<0.01)$.

\section{Discussion}

RA is a systemic and chronic autoimmune disease which 

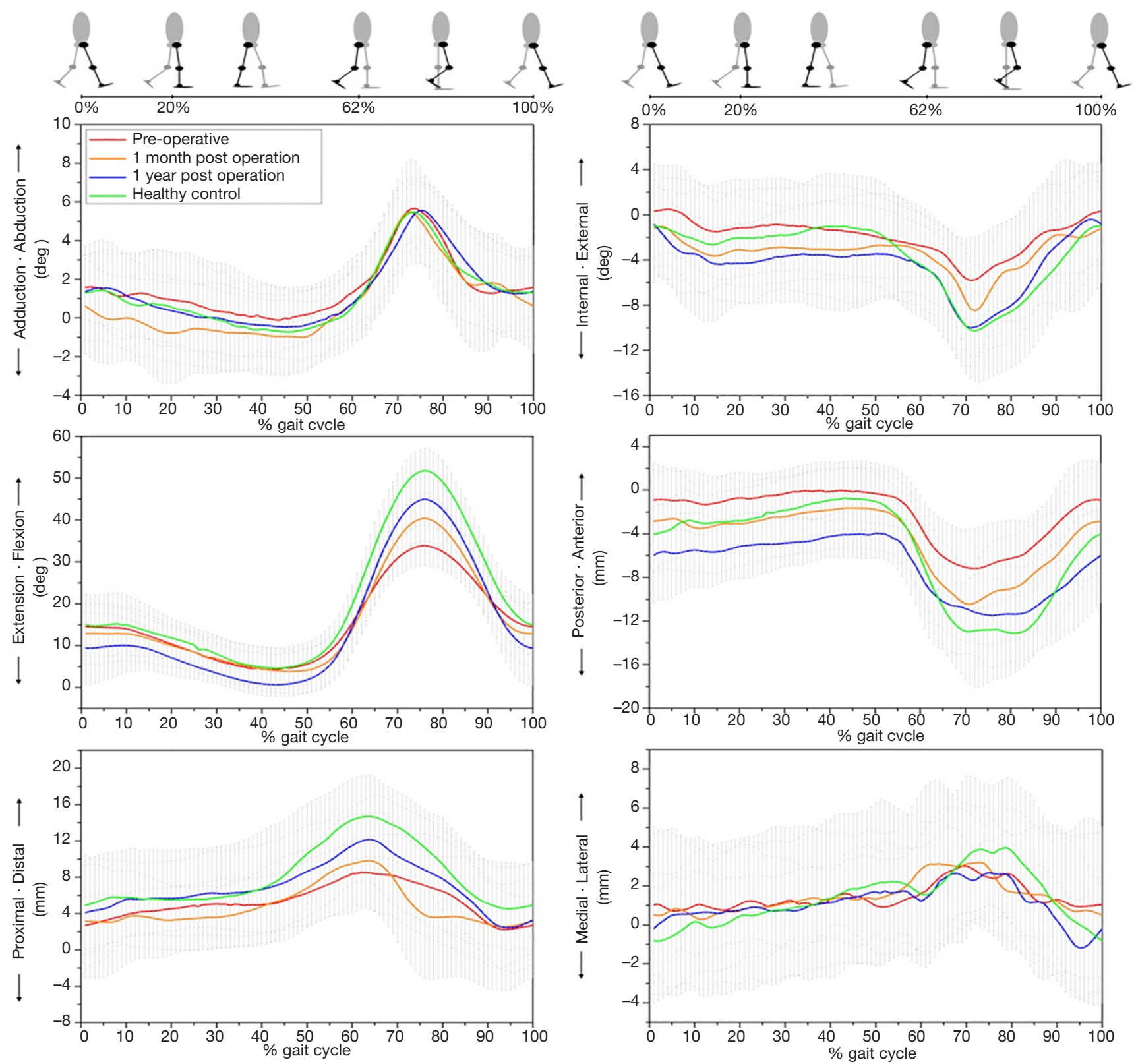

Figure 3 Tibiofemoral kinematics of the subjects in different groups during gait analysis. The thick solid lines represent the participants' average kinematics of the femur relative to the tibia and the gray shadow area represents the standard deviation of the participants. The green, red, yellow and blue lines represent the average motion of healthy, pre-operative, 1 month post operative and 1 year post operative, respectively. The gait begins with the heel strike and ends with the next heel strike. The gait cycle is divided into a stance phase and a swing phase by toe off (the vertical dashed line, about $62 \%$ gait). 

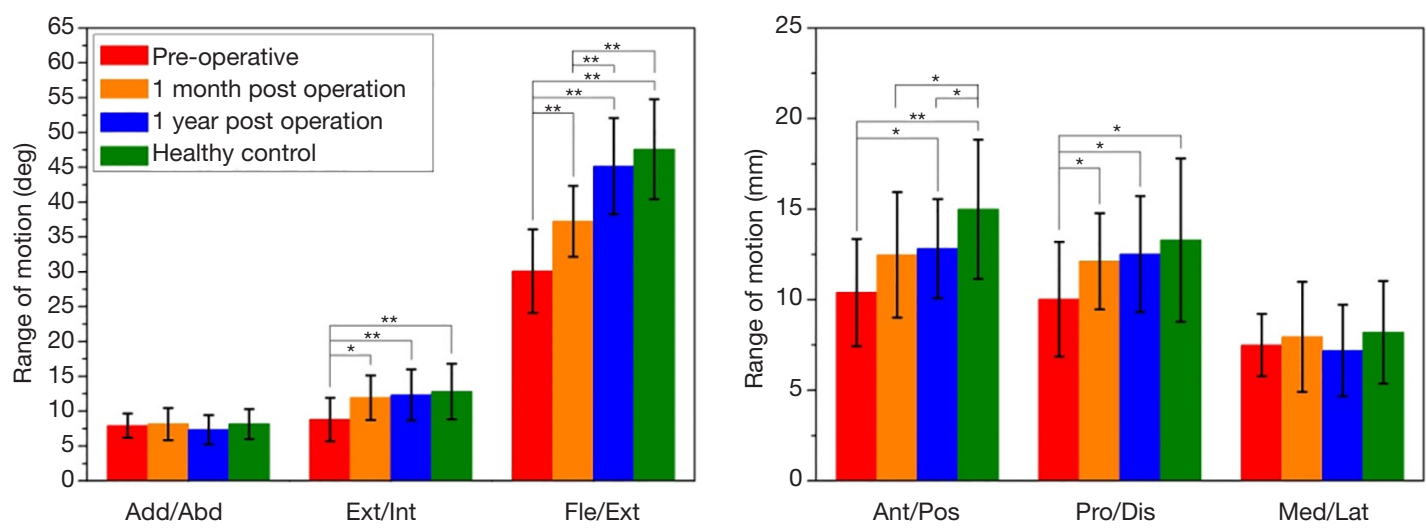

Figure 4 The range of motion of different groups. The green, red, yellow and blue bars represent the average motion of the Healthy, Preoperative, 1 month post operative and 1 year post operative, respectively. The symbol "** and “**” represent a significant difference between groups $(\mathrm{P}<0.05$ or $\mathrm{P}<0.01)$.

features the major pathological changes of symmetric, progressive, and corrosive arthritis. Its main clinical manifestations include red swollen patches, pain with heat in the joints, and morning stiffness. In the later stages, joint deformity and rigidity may occur which finally lead to disordered joint movement and cessation of function. The impact of RA on daily life is severe. Therefore, this disease has been studied closely by rheumatologists in China and over the world. The etiology and pathogenesis of RA have not yet been fully explained, and its treatment has continually been a critical and difficult issue in the field of rheumatology.

The current treatment for RA is predominantly medical treatment, mostly with slow acting anti-rheumatic drugs (SAARDs), non-steroidal anti-inflammatory drugs (NSAIDs), biological agents, biological therapy, and traditional Chinese medical therapies which have reported some clinically curative effects. However, these therapies are slow to take effect, have substantial side effects, and the medical treatment cost is high. Additionally, they have minimal effects in reducing the symptoms of local joint swelling and pain, and no effect on improving joint dysfunction. Chinese medicine categorizes RA in the scope of arthralgia syndromes and diseases of meridian-muscle regions. It is said in the book Natural Question: Artbralgia Theory that the 3 evils of wind, cold, and dampness commingle to become numbness, and that when numbness lodges in the muscles and bones, people feel pain in the joints. Arthralgia syndromes have a close connection with dysfunction of the meridian sinews. Meridian-sinews are the shortened form of the twelve main meridians which primarily encompass the bones and benefit the physiological functions of the organs. Normally functioning tendons are flexible, freely dredged, and accommodate healthy joint functions. Meridian-sinews that have been invaded by pernicious wind-cold-damp become blocked with the stagnation of qi and blood. This stagnation is the key reason for prolonged unhealed arthralgia syndromes. Therefore, dredging the meridian sinews is an effective method to treat arthralgia syndromes. In accordance with the meridian and tendon theory in Huangdi's Orthodox Classic: Natural Question, Professor Song Wei of the Department of Traditional Chinese Medicine, Southern War Zone General Hospital instigated the minimally invasive needle-knife scope technology to effectively address the drawbacks of the existing drug treatments for RA. Under visual observation, minimally invasive needle-knife scope technology utilizes self-developed, specialized devices to dredge the $\mathrm{j}$ tendons around the joint, restore the flow of qi and blood, and eliminate transversal lesions formed by phlegm and stagnation. This process is accompanied by the lavaging of the joints to clear away accumulated inflammatory factors and their immune complexes in the synovia, repair the articular surface, eliminate inflammation, remove adhesions, and restore joint function to achieve the fundamental objective of treating RA.

So far, researchers have applied the minimally invasive needle-knife scope in treating RA of the knee joint and follow-up assessments have found that the patients experienced reduced pain following treatment with remarkably improved knee function scores $(11,12)$. However, the research indicators used for these assessments 
were mainly subjective and are easily influenced by external factors, which resulted in great variance in the results reported. The evolution of gait analysis technology allows for gait to be precisely and quantitatively measured, and thus presents a brand new method to assess knee joint function.

Walking gait is a significant component of people's overall functional movement. When a healthy adult walk, the lower limbs swing in regular and rhythmic cycles, periodically and alternatively with approximately equal stride length and frequency. Though gait patterns vary with people's individual walking styles, the regularity and periodicity of walking is traceable. In walking, the interval between a heel contacting the ground and that same heel contacting the ground again is regarded as a walk cycle. In a gait cycle, each lower limb shall go through the two stages of standing on the ground and swinging off the ground, i.e., the stance phase (normally taking up $60 \%$ of the entire gait cycle) and the swing phase (normally taking up $40 \%$ of the entire gait cycle). According to the state of one-foot touching down, and then both feet touching down, the stance phase is divided into the single limb weight-bearing phase and the weight-bearing phase of both limbs $(1,13,14)$. The stance phase includes the whole process of the heel contacting the ground to the toe of the same side lifting off the ground. The specific movements include one lower limb stepping forward, its heel contacting the ground, then the entire foot contacting the ground with the center of balance immediately switching to that same side $(15,16)$.

It was revealed, through gait analysis, that after the minimally invasive needle-knife scope treatment was applied, during the end of stance phase, early and middle stages of swing phase (53-86\% of the gait cycle), extra extraversion appeared (approximately $5^{\circ}-7^{\circ}$ ). In addition, after 1 month of the treatment, at the middle and final stages of the stance phase (13-64\% of the gait cycle) the angle of tibial outward rotation increased by $6^{\circ}-9^{\circ}$. It can thus be shown that stripping of adhesions and loosening of knee joint tissues, synovial tissue planning, and articular cartilage surface repair with the minimally invasive needleknife scope had changed the contact area of the tibiofemoral joint. We can speculate that this is the major reason for apparent pain relief in knee joints following minimally invasive needle-knife scope treatment. However, we found that in the stance phase and initial swing phase, the patients showed greater ROMs in knee joint inward and outward rotation, upward and downward movement, plus flexion and extension after 1 year of having finished the treatment as compared with just 1 month of the therapy. In motion, the stage from a heel contacting the ground to mid-stance phase ( $0-53 \%$ of the gait cycle) and swing phase $(63-100 \%$ of the gait cycle) saw a distal femoral shift (approximately 3-9 mm) relative to the tibia. The enlarged space between the femur and tibia may reduce the contact pressure between them and relieve pain. Meanwhile, compared with the kinematic data preceding treatment, increased abduction, femoral rotation, and remote movement were found at the 1 year follow up of the treatment. These changes were achieved by lavage within the joint, which provoked the clearance of hyperplastic hypertrophic synovium and osteophytes, articular surface repair, removal of the bulk of inflammatory factors in the synovial fluids to eliminate local inflammation and reduce inflammatory substances in response organisms in the joint. Lavage also promoted the timely clearance of fibronectin, cellulose, and immune complexes in the target articular cartilages, synovium, and joint fluids to block localized immune disorders, which in turn benefited articular cartilage nutrition and regeneration, promoting joint function recovery and remarkably enhancing the functional recovery of knee joints. Visually guided loosening treatment can strip the surrounding diseased soft tissues, relieve spasms of the ligaments and tendons, and effectively adjust joint space to improve joint motion (17). Mechanical stimulation and tendon loosening under needle-knife scope has a functional mechanism which may enhance the capillary and lymphatic circulation of local tissues and organs, improve the ability to metabolize diseased tissues, enlarge interstitial fluid channels, improve metabolism of masses and reduce pressure from edema, promote the absorption of diseased tissues substances, and lesions which accelerates recovery (18). The major limitation of this study, being a pilot case study, was the small sample size. We plan to undertake further controlled studies of RA patients with a larger sample size.

\section{Conclusions}

This experimental study indicates improvements in knee joint functions of RA patients, which demonstrates promising effects of minimally invasive needle-knife scope therapy in treating RA of the knee joint. Moreover, for RA of the knee joint occurring in different compartments, gait analysis can reveal the changes in moment of impact and surface electromyogram resulting from the treatment, which is a significant method of assessment of knee joint function for the future, and a potential reliable supplement 
for pain and function scores.

\section{Acknowledgments}

The authors herein would like to extend their immense gratitude to Dr. Yu Zhang for his technical support and Guangzhou Infumedi Medical Technology Co., Ltd. for their generous instrumental assistance.

Funding: This study was partially funded by the National Natural Science Foundation of China (Project Number: 81054276), Guangdong science and Technology Planning Projects (Project Number: 2014A020221119, 2015B020233011), Guangzhou Science and Technology Planning Projects (Project Number: 201704020160) and theMilitary Logistics Research Project (Project Number: CGZ16C007, GLB18J039).

\section{Footnote}

Reporting Checklist: The authors have completed the STROBE reporting checklist. Available at http://dx.doi. org/10.21037/apm-20-1705

Data Sharing Statement: Available at http://dx.doi. org/10.21037/apm-20-1705

Conflicts of Interest: All authors have completed the ICMJE uniform disclosure form (available at http://dx.doi. org/10.21037/apm-20-1705). The authors have no conflicts of interest to declare.

Ethical Statement: The authors are accountable for all aspects of the work in ensuring that questions related to the accuracy or integrity of any part of the work are appropriately investigated and resolved. The study was approved by the institutional research ethics committee of the Southern War Zone General Hospital and written informed consent was obtained from all participants. All procedures performed in this study involving human participants were in accordance with the Declaration of Helsinki (as revised in 2013).

Open Access Statement: This is an Open Access article distributed in accordance with the Creative Commons Attribution-NonCommercial-NoDerivs 4.0 International License (CC BY-NC-ND 4.0), which permits the noncommercial replication and distribution of the article with the strict proviso that no changes or edits are made and the original work is properly cited (including links to both the formal publication through the relevant DOI and the license). See: https://creativecommons.org/licenses/by-nc-nd/4.0/.

\section{References}

1. Fransen J, van Riel PL. The Disease Activity Score and the EULAR response criteria. Clin Exp Rheumatol 2005;23:S93-9.

2. Arnett FC, Edworthy SM, Bloch BA, et al. The American Rheumatism Association 1987 revised criteria for the classification of rheumatoid arthritis. Arthritis Rheum 1988;31:315-24.

3. Abuwarwar MH, Knoblich K, Fletcher AL. A pathogenic hierarchy for synovial fibroblasts in rheumatoid arthritis. Ann Transl Med 2018;6:S75.

4. Ospelt C, Neidhart M, Gay RE, Gay S. Synovial activation in rheumatoid arthritis. Front Biosci 2004;9:2323-34.

5. Wei S, Sun W, Chen Z. 121 Case Analysis for Traditional Chinese Medical Minimally Invasive Needle Scope Application in Local Intractable Swellings of Arthromyodynia.Chinese Journal of Misdiagnostics 2011;11:2188-9.

6. Wei S, Sun W, Chen Z. Minimally Invasive Needle ScopeTreatment on 40 Cases of RheumatoidArthritis at Active Stage. Chinese Journal of Clinical Healthcare 2011;14:413-5.

7. Chen Z, Wei S, Sun W.Clinical Therapeutic Effect Evaluation for Transverse Venation Unhitching Treatment on RheumatoidArthritis at Active Stage. Chinese Journal of Clinical Healthcare 2011;14:123-6.

8. Wei S, Li N, Chen Z, et al. Clinical Observation of Transverse Venation Unhitching Treatment on RheumatoidArthritis. Chinese Medicine Emergency 2009;18:537-8.

9. Pincus T, Sokka T. Quantitative measures and indices to assess rheumatoid arthritis in clinical trials and clinical care. Rheum Dis Clin North Am 2004,30:725-51.

10. Carlin JB, Doyle LW. Statistics for clinicians: 7: Sample size. J Paediatr Child Health 2002;38:300-4.

11. Wei S, Qiu Y, Chen Z, et al. Minimally invasive expansion lysis treatment of supraspinatus tendinitis. Chinese Journal of Orthopedics and Traumatology of Chinese Medicine 2007;15:35.

12. Chen Z, Wei S, Sun W. Clinical observation of 80 cases of knee osteoarthritis treated with traditional Fasciameridianscope. Chinese Journal of Guiyang College of Traditional Chinese Medicine 2011;22:56-7. 
13. Xue L. China Fascia-meridian learning. Beijing: Publishing House of Ancient Books of China, 2009.

14. Tan $\mathrm{Y}$, Zheng $\mathrm{Y}$, Zhan $\mathrm{H}$, et al. Three-Dimensional Gait Analysis Application in Knee Osteoarthritis Study. International Journal of Orthopaedics 2014;35:215-8.

15. Springer S, Yogev Seligmann G. Validity of the Kinect for Gait Assessment: A Focused Review. Sensors (Basel) 2016;16:194.

16. Asay JL, Boyer KA, Andriacchi TP. Repeatability of gait analysis for measuring knee osteoarthritis pain

Cite this article as: Chen Z, Wei S, Xu W, Li X, Zhang X, Liu $\mathrm{Y}, \mathrm{Li} \mathrm{H}$, Chen J. Adoption of a new gait system to evaluate the clinical effects of minimally invasive needle-knife scope therapy for the treatment of rheumatoid arthritis of the knee joint. Ann Palliat Med 2020;9(5):3340-3349. doi: 10.21037/apm-20-1705 in patients with severe chronic pain. J Orthop Res 2013;31:1007-12.

17. Fife RS, Braunstein EM. Relationship between arthroscopic evidence of cartilage damage and radiographic evidence of joint space narrowing in early osteoarthritis of the knee. Arthritis Rheum 1991;34:377-82.

18. Liu B. Acupotomy. Clinical Medicine of China 2000;28:2 .

(English Language Editor: J. Jones) 